\title{
La encefalopatía hiperamonémica, una posible complicación por derivación urinaria tras cistectomía radical. Revisión de la literatura a proposito de un caso
}

\author{
Pérez-Fidalgo JA*, Chirivella González I*, Günthner S**, Cervera Miguel JI*, March Villalba JA**, \\ Cervantes Ruipérez A.*
}

*Servicios de Oncología Médica y ${ }^{* *}$ Urología. Hospital Clinico Universitario. Valencia.

Actas Urol Esp. 2007;31(4):394-399

\section{RESUMEN}

LA ENCEFALOPATÍA HIPERAMONÉMICA, UNA POSIBLE COMPLICACIÓN POR DERIVACIÓN URINARIA TRAS CISTECTOMÍA RADICAL. REVISIÓN DE LA LITERATURA A PROPOSITO DE UN CASO

Introducción: La cistectomía radical es una intervención con una elevada morbilidad. Las derivaciones postcistectomía pueden ocasionar complicaciones metabólicas como la encefalopatía hiperamonémica.

Caso Clínico: Presentamos el caso de una paciente cistectomizada por un carcinoma vesical con posterior ureterosigmoidostomía. Tras la intervención acudió en 4 ocasiones con síntomas neurológicos y confusión. Todo el estudio analítico y pruebas de imagen fueron normales. Los niveles de amonio en sangre estaban elevados. Tras tratamiento de soporte y hemodiálisis la clínica desapareció. Posteriormente fue sometida a una nueva derivación quirúrgica (conducto ileal).

Discusión: El empleo de técnicas de derivación continentes tras la cistectomía se aconseja ante la importante afectación de la calidad de vida que produce el conducto ileal. Sin embargo, estas técnicas tienen complicaciones, como la encefalopatía hiperamonémica de causa no hepática. La elevada absorción de amonio por el epitelio intestinal de la neovejiga induce una encefalopatía. Es esencial su diagnóstico temprano con el fin de iniciar un tratamiento eficaz.

Palabras clave: Hiperamonemia. Cistectomia radical. Ureterosigmoidostomia.

\section{ABSTRACT \\ HYPERAMMONAEMIC ENCEPHALOPATHY, POSSIBLE COMPLICATION AFTER URINARY DIVERSION IN RADICAL CYSTECTOMY. REVIEW OF THE LITERATURE WITH REGARD TO A CLINICAL CASE}

Introduction: Radical cystectomy is an intervention with an important morbidity. Urinary diversion is a possible cause of metabolic complications like hyperammonaemic encephalopathy.

Case report: We present the case of a patient treated with a radical cystectomy and modified ureterosigmoidostomy after diagnosis of bladder cancer. After surgery the patient presented in 4 ocasions recurrent episodic confusion. Laboratory and image tests were normal. Levels of seric ammonium was increased. After supportive treatment and hemodyalisis symptoms disappeared. Later patient was reoperated and a reconstruction to ileal conduit was made.

Discussion: Continent urinary diversions are advised due to important negative impact on quality of life produced by ileal conduit. However these diversions have several complications, like encephalopathy secondary to non-hepatic hyperammonaemia. Increased absortion of ammonium by intestinal tissue of the new-ladder induces encephalopathy. Early diagnosis of this complication is essencial in order to administer an effective treatment. 
$\mathrm{E}$ tratamiento estándar del cáncer infiltrante de vejiga es la cistectomía radical $^{1}$. Esta intervención tiene una elevada morbilidad derivada no sólo de la importante resección de estructuras sino también del tipo de derivación urinaria empleada tras la extirpación de la vejiga. La mayor parte de las complicaciones postcistectomía suelen ocurrir en el postoperatorio inmediato. Sin embargo, un pequeño porcentaje de pacientes presenta además otras complicaciones de instauración subaguda, algunas de tipo metabólico, como la hiperamonemia ${ }^{2}$.

Los elevados niveles de amonio en sangre producen una encefalopatía caracterizada por episodios confusionales que puede llegar incluso provocar el coma. La causa más frecuente de hiperamonemia es la descompensación hepática en el contexto de una hepatopatía crónica siendo muy infrecuentes las secundarias a derivaciones urinarias.

Hemos realizado una revisión de la literatura sobre la encefalopatía hiperamonémica no hepática como complicación metabólica tras una derivación urinaria, a propósito del caso de una paciente que acudió a nuestro hospital después de haber sido intervenida de un carcinoma vesical mediante cistectomía radical con ureterosigmoidostomía tipo Mainz II.

\section{CASO CLÍNICO}

Se trata de una mujer de 66 años diabética y con antecedentes de hepatitis por virus $\mathrm{C}$ de etiologia postransfusional.

En Enero del 2006 presentaba un síndrome miccional pertinaz que no respondía a tratamiento por lo que se le realizó una cistoscopia con posterior RTU. Fue diagnosticada de un carcinoma vesical pTa G3 tras lo que inició tratamiento con BCG intravesical.

La RTU de control tras finalizar el tratamiento con BCG detectó presencia de múltiples focos de carcinoma en la vejiga confirmándose el diagnóstico de un carcinoma urotelial que invadía la capa muscular (pT2 G3).

En Julio del 2006 es sometida a una cistectomía radical con derivación urinaria mediante ureterosigmoidostomía tipo Mainz II. La anatomía patológica demostró un carcinoma urotelial de alto grado con invasión de capa muscular y con afectación de uno de los ganglios linfáticos resecados, se trataba de un pT2a N1. No se detectaron metástasis a distancia.

Dos semanas después de la intervención, acudió con desorientación y tendencia al sueño en el contexto de un cuadro compatible con infección de vías urinarias. Tras 24 horas en observación, con hidratación intravenosa y antibioterapia se recuperó y pudo ser dada de alta con antibiótico oral.

Diez días después acudió de nuevo al hospital por aparición de dispraxia, tendencia al sueño, lenguaje incoherente y obnubilación. Durante el episodio presenta además un pico febril de $38,5^{\circ} \mathrm{C}$. La bioquímica y el hemograma eran normales (sin leucocitosis ni neutrofilia). Se realizó una ecografía abdominal que identificó una hidronefrosis bilateral moderada y un TAC cerebral que fue normal.

Durante su ingreso, y tras inicio de tratamiento antibiótico de amplio espectro y fluidoterapia, el cuadro confusional fue solucionándose hasta una recuperación completa.

A los pocos días, la paciente volvió a presentar deterioro cognitivo. En esta ocasión el inicio de los síntomas centrales había sido similar, con dispraxia, dificultad repentina para realizar tareas cotidianas, somnolencia y obnubilación, sin embargo en esta ocasión acudió al hospital en situación de coma, con un Glasgow de 8. Estaba afebril.

En la analítica destacaba únicamente una moderada hiperglucemia y una ligera elevación de transaminasas.

Se le realizó un electroencefalograma en el que se observaban unas ondas lentas sobre áreas de ambos hemisferios, hallazgos característicos de una encefalopatía metabólica. Un TAC cerebral no mostró anomalías estructurales. De forma similar al ingreso previo, tras varios días hospitalizada con fluidoterapia de soporte y dieta absoluta, la paciente experimenta una progresiva mejoría con recuperación completa de la clínica.

Tres semanas después, la paciente vuelve a notar síntomas dispraxia y tendencia al sueño de instauración progresiva. Acude a urgencias donde es evaluada sin encontrarse alteraciones en la exploración ni en las pruebas analíticas ni de imagen por lo que se le remite a domicilio, no 
existía fiebre ni ninguna clínica sugestiva de infección. A las 24 horas acude con una alteración severa del nivel de conciencia con un Glasgow de 6 .

En esta ocasión la paciente acudía tras haber iniciado 15 días antes, un primer ciclo de quimioterapia adyuvante con esquema taxol 175 $\mathrm{mg} / \mathrm{m} 2$ y carboplatino AUC 5. Por ello, presentaba una leucopenia con neutropenia atribuible a una mielotoxicidad postquimioterapia. Todos los demás parámetros electrolíticos, incluyendo calcemia, eran rigurosamente normales. La gasometría venosa demostraba un $\mathrm{pH}$ de 7.44. El TAC cerebral y la radiografía de tórax fueron normales.

Ante la presencia de episodios repetidos de síndrome confusional de probable causa metabólica, todos ellos en 3 meses desde la cistectomía, se consideró que podría ser una complicación metabólica de la derivación. Tras haberse descartado que se tratara de una acidosis metabólica se decidió solicitar unos niveles de amonio en sangre, que demostraron una amonemia de 400 microgramos/dl (normal entre 17-80).

Ante el diagnóstico de encefalopatía hiperamonémica de causa no-hepática se inició fluidoterapia, dieta absoluta, enemas con lactulosa y ante la gravedad clínica se inició hemodiálisis. Tras 3 sesiones de hemodiálisis la paciente experimentó una recuperación neurológica paralela a la corrección de los niveles de amonio que disminuyeron hasta normalizarse.

Posteriormente se realizó una reconversión quirúrgica de la ureterosigmoidostomía a conducto ileal. Actualmente la paciente continúa con el tratamiento quimioterápico adyuvante y no ha vuelto a presentar nuevos episodios confusionales.

\section{DISCUSIÓN}

La cistectomía radical continúa siendo el tratamiento de elección en el manejo de los carcinomas uroteliales infiltrantes. Esta intervención consiste en la resección de la vejiga, linfadenectomía pélvica bilateral, resección de grasa perivesical y de porción distal de ambos uréteres y proximal de la uretra. En hombres se realiza prostatectomía y resección de vesículas seminales y en mujeres histerectomía, doble anexectomía y resección de parte de vagina.
Tras la cistectomía, el reto al que se debe de enfrentar el urólogo, es la selección de la derivación urinaria más apropiada. Se han descrito múltiples derivaciones urinarias en la literatura (Tabla 1). Estas derivaciones se clasifican según su grado de funcionalidad en incontinentes, y continentes subdividiéndose estas últimas a su vez en reservorios cutáneos y en neovejigas ortotópicas ${ }^{3}$. La técnica incontinente más extendida es el conducto ileal que es un método simple y seguro de derivar la orina pero mediante un drenaje continuo a través de un estoma que afecta negativamente a la calidad de vida del paciente. Para intentar evitar el impacto negativo del estoma se desarrollaron múltiples técnicas con intención de mantener la continencia, pues esto induciría una mejoría de la calidad de vida. Sin embargo aunque en algunos estudios las técnicas continentes parecen demostrar un impacto positivo en la calidad de vida ${ }^{4}$, en otros no se consigue demostrar claramente que exista tal beneficio frente al conducto ileal ${ }^{5}$. Todas las técnicas quirúrgicas empleadas tienen sus ventajas y desventajas lo cual debe ser tomado en consideración en el momento de decidir el tipo de derivación a realizar.

La agresividad de la cistectomía y la derivación urinaria empleada provocan una morbilidad cercana al 30\% y una mortalidad de $1-3 \%{ }^{6}$. Las

\section{Tabla 1}

Tipos de derivaciones urinarias en pacientes cistectomizados

\begin{tabular}{lc}
\hline Derivación urinaria & $\begin{array}{c}\text { Segmento intestinal } \\
\text { empleado }\end{array}$ \\
\hline $\begin{array}{l}\text { Derivaciones incontinentes } \\
\text { Conducto ileal }\end{array}$ & Ileon \\
Conducto colónico & Colon \\
Urostomía cutánea & Ninguno \\
Reservorios cutáneos (continentes) & \\
Tipo Florida & Ileocecal \\
Tipo Indiana & Ileocecal \\
Tipo Koch & Ileon \\
Tipo Mainz I & Ileocecal \\
Neovejigas ortotópicas (continentes) & \\
Hautman & Ileon \\
Studer & Ileon \\
Bolsa en T & Ileon \\
Ureterosigmoidostomía & \\
Mainz tipo II (continente) & Sigma \\
\hline
\end{tabular}


complicaciones postoperatorias tanto inmediatas como tardías son pues relativamente frecuentes (Tabla 2). Una de estas complicaciones son las encefalopatías metabólicas, éstas suelen ser debidas a que los segmentos empleados en la derivación, ya sea colon o ileon, mantienen su capacidad absortiva y secretora. Tanto ileon como colon secretan sodio y bicarbonato y reabsorben amonio, iones de hidrógeno y cloro. Esto puede inducir una acidosis metabólica hiperclorémica y estados de hiperamonemia ${ }^{7}$. El desarrollo de una hiperamonemia de causa no hepática en pacientes sometidos a derivación urinaria obedece al prolongado contacto de la orina con el epitelio intestinal, lo que motiva la absorción de elevadas concentraciones de sustancias excretadas vía urinaria como el amonio. Esto motivaría una saturación de la capacidad metabolizadora del hígado y por lo tanto una elevada liberación de amonio al torrente sanguíneo (Fig. 1).

Aunque la causa más frecuente de elevación de la amonemia es la hepatopatía crónica, la presentación clínica de la hiperamonemia no hepática es variable y con frecuencia episódica. El cortejo de síntomas incluye alteraciones de la personalidad, confusión, irritabilidad y ataxia. Las náuseas, la letargia y la hiperventilación también pueden estar presentes ${ }^{8,9}$. En casos graves puede llegar al coma. No obstante la encefalopatía severa es más frecuente en aquellos pacientes que asocien cierto deterioro de la función hepática, presentándose en éstos dos mecanismos patogénicos, la alteración hepática y el incremento en la absorción de amonio. Este podría ser el caso de nuestra paciente, en la que además de la ureterosigmoidostomía padecía una hepatopatía por virus C.

En el caso de la encefalopatía hiperamonémica asociada a derivaciones urinarias, es frecuente que la clí-
Tabla 2

Complicaciones de la cistectomía radical.

\begin{tabular}{cc}
\hline Complicaciones & $\begin{array}{c}\text { Incidencia } \\
\text { aproximada }\end{array}$ \\
\hline Menores & $12-20 \%$ \\
Ileo paralítico & $2-7 \%$ \\
Infección de la herida & $2 \%$ \\
Neumonía & $2 \%$ \\
Encefalopatía metabólica & $1-3 \%$ \\
Infección de tracto urinario & $1 \%$ \\
Insuficiencia renal aguda & $1 \%$ \\
Arritmia cardiaca & $1 \%$ \\
Trombosis venosa profunda & $1 \%$ \\
Daño rectal intraoperatorio & $1 \%$ \\
Dehiscencia & \\
& $2 \%$ \\
Reintervención & $1 \%$ \\
Accidente cerebrovascular & $1 \%$ \\
Sepsis & $1 \%$ \\
Fallo respiratorio & $1 \%$ \\
Tromboembolismo pulmonar & $1 \%$ \\
Infarto de miocardio & $1-3 \%$ \\
\hline
\end{tabular}

nica neurológica aparezca de forma recurrente tras la recuperación clínica de un episodio ${ }^{10}$, del mismo modo que en nuestro caso en el que se produjeron hasta 4 cuadros confusionales recurrentes. La variabilidad clínica y la infrecuente

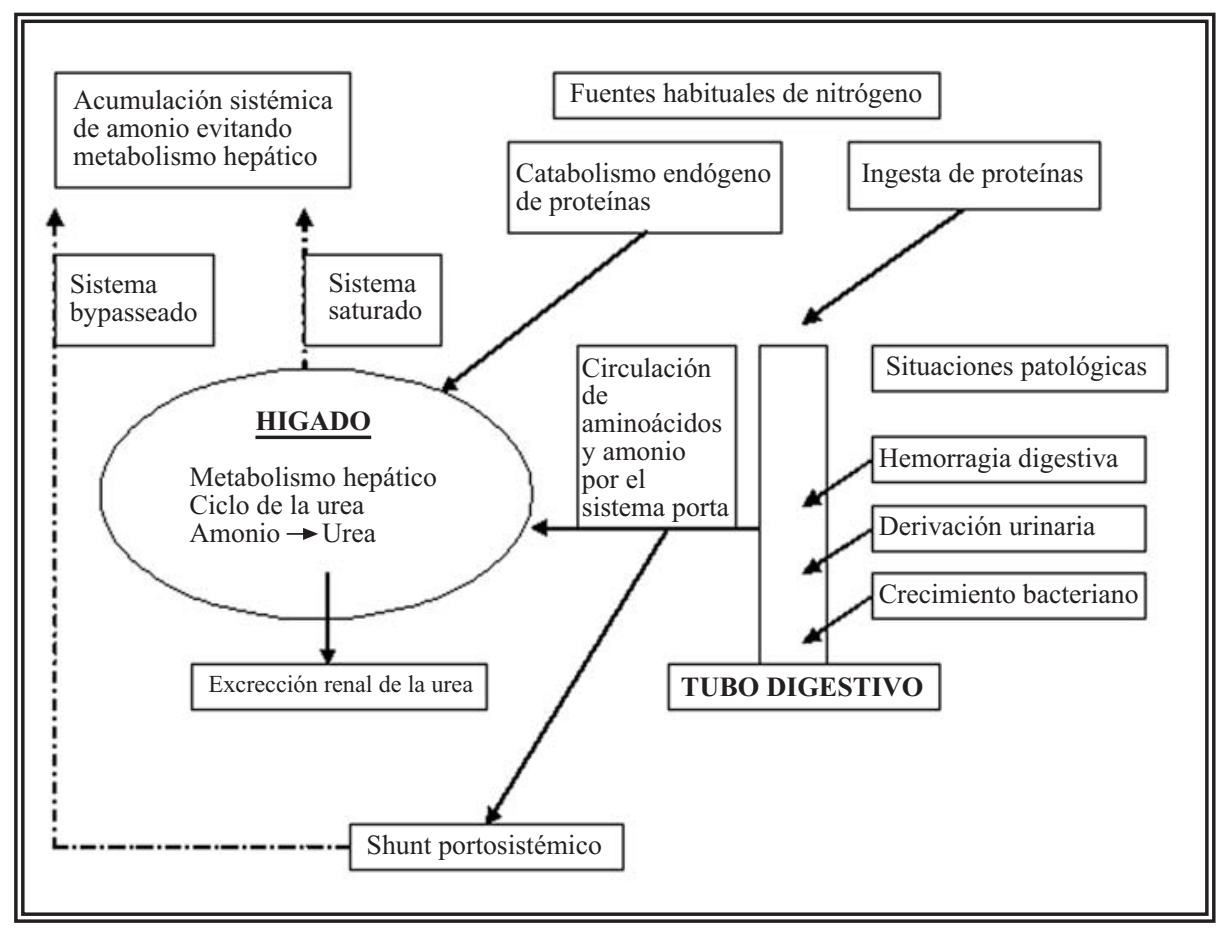

FIGURA 1. Representación de las fuentes de producción de amonio y sus vias de excrección - tomado de Hawkes et $\mathrm{al}^{2}$. 
presentación hacen que su diagnóstico sea frecuentemente demorado. En estos casos es de vital importancia el reconocimiento en la historia clínica de factores que podrían predisponer o causar la acumulación de amonio. Estos factores, aparte de la hepatopatía crónica, pueden ser muy variados (Tabla 3 ).

Tabla 3

Causas de hiperamonemia no hepática

\section{Causas de hiperamonemia no hepática}

Defectos congénitos de enzimas del ciclo de la urea

Defectos en el transporte de intermediarios del ciclo de la urea

Otras causas metabólicas:

- Hipoglucemia hiperinsulinémica

- Acidosis tubular distal

- Déficit primario de carnitina

- Defectos de oxidación de los ácidos grasos

Farmacológico:

- Quimioterapia: 5FU, asparraginasa.

- Acido valproico

- Agentes anestésicos

Sindrome de Reye

Shunt portosistémico

Urinaria:

- Derivaciones urinarias: como la ureterosigmoidostomía

- Infecciones urinarias

- Reflujo vesico-ureteral

Hematológicas:

- Mieloma múltiple

- Algunas leucemias

Otras causas:

- Nutrición parenteral

- Idiomática

La medición de los niveles de amonio en sangre constituye la clave para la realización de un diagnóstico temprano. A pesar de que los niveles suelen ser superiores a $150 \mu \mathrm{g} / \mathrm{dl}$, no siempre existe una correlación clínica entre el grado de encefalopatìa y los niveles de amonio. Es aconsejable descartar disfunción hepática asociada y alcalosis hiperclorémica en el caso de hiperamonemia asociada a derivación urinaria. El electroencefalograma puede detectar una encefalitis difusa inespecífica. El TAC y la resonancia cerebral pueden detectar ocasionalmente un cierto grado de edema cerebral.
La patogenia de la encefalopatía asociada al amoniaco todavía no está bien establecida. Uno de los efectos atribuidos al amonio es la inhibición de la enzima a-ketoglutarato deshidrogenasa lo cual favorece la formación de glutamina a partir de a-ketoglutarato. Todos estos cambios producirán una proteolisis que induciría la encefalopatía $^{11,12}$.

Antes del tratamiento, determinar la causa es fundamental, ya que la corrección de la situación desencadenante permite evitar nuevos episodios y a veces la corrección definitiva. El tratamiento del episodio agudo en la hiperamonemia no hepática no está claramente establecido. Al igual que en la encefalopatía hepática en estos casos se recomienda la disminución de los niveles de amonio en luz intestinal. Las medidas más empleadas son la reducción de la ingesta de proteínas en la dieta a 20 gr de proteínas al día y el tratamiento con disacáridos no absorbibles como la lactulosa y el lactitiol con el fin de favorecer la eliminación intestinal del amonio ${ }^{13}$. La administración de sueros glucosados al 10 o $20 \%$ disminuye la absorción de aminoácidos en el tubo digestivo.

La antibioterapia oral o intravenosa favorece el control sintomático en aquellos casos en los que existe un sobrecrecimiento bacteriano con sobreproducción de urea. En pacientes con alteraciones del metabolismo de la urea se han empleado el sodio benzoato o sodio fenilacetato, en aquellos casos con déficit de ornitina transcarbamilasa.

En casos severos un tratamiento de soporte adecuado incluyendo ventilación en caso de desarrollo de coma puede llegar a ser necesario. Si no existe una pronta respuesta al tratamiento o si existe gravedad clínica la hemodiálisis ha demostrado ser eficaz ${ }^{14}$.

En resumen, la hiperamonemia no hepática supone un reto para el clínico por su baja frecuencia y la variabilidad de su presentación clínica. La sintomatología neurológica no explicada en un paciente con antecedentes como la derivación urinaria debe de ser la base para un diagnóstico de sospecha.

\section{REFERENCIAS}

1. Carrion R, Seigne J. Surgical management of bladder carcinoma. Cancer Control. 2002;9(4):284-292. 
2. Hawkes ND, Thomas GA, Jurewicz A, Williams OM, Hillier CM, McQueen IN, et al. Non-hepatic hyperammonaemia: an important potentially reversible cause of encephalopathy. Postgrad Med. J. 2001;77:717-722.

3. García de Jalón Martínez A., Sancho Serrano C., Trívez Poned M.A., Valdivia Navarro P., Gonzalvo Ibarra A. Roncalés Badal A. et al. Urinary diversions and bladder enlargements Actas Urol Esp. 2002;26(7):467-480.

4. Conde Redondo C, Estebanez Zarranz J, Rodriguez Tovez A, Amon Sesmero J, Alonso Fernandez D, Martinez Sagarra JM. Quality of life in patients treated with orthotopic bladder substitution versus cutaneous ileostomy. Actas Urol Esp 2001;25(6):435-444.

5. Gerharz EW, Mansson A, Hunt S, Skinner EC, Mansson W. Quality of life after cystectomy and urinary diversion: an evidence based analysis. J Urol. 2005;174(5):1729-1736.

6. Sullivan JW, Montie JE. Summary of complications of ureteroileal conduit with radical cystectomy : review of 336 cases. Semin Urol Oncol. 1997;15:94-98.

7. Mundy AR. Metabolic complications of urinary diversion. Lancet. 1999;353(9167):1813-1814.

8. Butterworth RF. Effects of hyperammonaemia on brain function. J Inherit Metab Dis. 1998;21(Suppl 1):6-20.

9. O`Neill M, Dubrey RW, Grocott-Mason RM. Valproate encephalopathy and hyperammonaemia. Postgrad Med J. 2002;78(919):316-317.
10. Oliver RM, Talbot S, Raman GV. Hyperammonaemic coma in ureterosigmoid urinary diversion. Postgrad Med J. 1989;65(765):502-504.

11. Ott P, Clemmesen O, Larsen FS. Cerebral metabolic disturbances in the brain during acute liver failure: from hyperammonaemia to energy failure and proteolysis. Neurochem Int. 2005;47(1-2):13-18.

12. Butterworth RF. Effects of hyperammonaemia on brain function. J Inherit Metab Dis 1998;21 Suppl 1:6-20.

13. Riordan SM, Williams R. Treatment of hepatic encephalopathy. N Eng J Med. 1997;337:473-479.

14. Wilson BE, Hobbs WN, Newmark JJ, Farrow SJ. Rapidly fatal hyperammonemic coma in adults-urea cycle enzyme deficiency. West J Med. 1994;161:166-168.

Correspondencia autor: Dr. J.A. Pérez-Fidalgo

Servicio de Oncología. Hospital Clínico Universitario.

Avda. Blasco Ibáñez, 17. 46010 Valencia.

Tel.: 963862600

E-mail autor: japfidalgo@msn.com

Información artículo: Revisión

Trabajo recibido: diciembre 2006

Trabajo aceptado: enero 2007 